\title{
BMJ Open Weight change, cardio-metabolic risk factors and cardiovascular incidence in people with serious mental illness: protocol of a population-based cohort study in the UK from 1998 to 2020
}

Charlotte Lee (D) ,1,2 Felicity Waite (D) ,3,4 Margaret C Smith (D) ,,2 Min Gao (D) ,,5 Clare Bankhead, ${ }^{1,2}$ Paul Aveyard (D) , ${ }^{1,2}$ Carmen Piernas (D) ${ }^{1}$

To cite: Lee C, Waite F, Smith MC, et al. Weight change, cardio-metabolic risk factors and cardiovascular incidence in people with serious mental illness: protocol of a populationbased cohort study in the UK from 1998 to 2020. BMJ Open 2021:11:e053427. doi:10.1136/ bmjopen-2021-053427

- Prepublication history and additional supplemental materia for this paper are available online. To view these files, please visit the journal online (http://dx.doi.org/10.1136/ bmjopen-2021-053427)

$\mathrm{PA}$ and $\mathrm{CP}$ contributed equally.

Received 17 May 2021 Accepted 11 October 2021

Check for updates

(c) Author(s) (or their employer(s)) 2021. Re-use permitted under CC BY. Published by BMJ.

${ }^{1}$ Nuffield Department of Primary Care Health Sciences, University of Oxford, Oxford, UK

${ }^{2} \mathrm{NIHR}$ Oxford Biomedical

Research Centre, Oxford University Hospitals NHS Foundation Trust, Oxford, UK ${ }^{3}$ Department of Psychiatry, University of Oxford, Oxford, UK ${ }^{4}$ Oxford Health NHS Foundation Trust, Oxford, UK

${ }^{5}$ School of Public Health, Peking University Health Science Centre, Beijing, China

Correspondence to

Charlotte Lee;

charlotte.lee@phc.ox.ac.uk

\section{ABSTRACT}

Introduction People with serious mental illness (SMI), which includes people with diagnoses of schizophrenia spectrum and bipolar disorders, face significant health inequality. This includes a life expectancy reduced by 1520 years mostly due to premature cardiovascular disease (CVD) compared with the general population. Excess weight gain and related comorbidities are preventable risk factors for CVD. To improve the understanding and management of CVD in people with SMI, we will examine the association between SMI and: (1) weight change; (2) cardio-metabolic risk factors for CVD; and (3) incidence of and mortality from CVD. We will also (4) examine the incidence of referral to weight management services for people with SMI compared with people without SMI. Methods and analysis In this retrospective cohort study, we will link general practice records from the UK Clinical Practice Research Datalink Aurum database. We will establish a cohort of patients diagnosed with SMI between 1998 and 2020 who are matched with up to four controls on age, sex, general practice and calendar year. We will use multivariable mixed-effects linear regression models and Cox proportional hazard models with sequential adjustment for potential confounders identified by separate directed acyclic graphs.

Ethics and dissemination This study has been reviewed and approved by the Independent Scientific Advisory Committee for Medicines and Healthcare products Regulatory Agency database research. The results will be published in a peer-reviewed journal.

\section{INTRODUCTION}

Serious mental illness (SMI) includes psychotic disorders like schizophrenia spectrum and bipolar disorders, which are marked by hearing, seeing, or believing things that are not real. ${ }^{1}$ People with SMI have a life expectancy that is approximately 15-20 years lower than the general population. ${ }^{2}$ Two-thirds of premature mortality in this group is explained by preventable cardiovascular disease (CVD). ${ }^{3}$ A 2017 meta-analysis

\section{Strengths and limitations of this study}

- The key strengths of the study include the representative population contained in the Clinical Practice Research Datalink Aurum, the sizeable and linked dataset, and substantial statistical power.

- However, limitations include its observational nature and potential for residual confounding due to lack of information on some factors.

- There is also potential for selection bias since physical health checks are not always performed on random population samples.

reported people with SMI have a significantly increased incidence of and mortality from than the general population: HR: $1.78,95 \%$ CI $1.60,1.98 .^{4}$ This indicates an important clinical need to identify the factors underlying the higher incidence of CVD for this group and routes for prevention and treatment. ${ }^{56}$

Overweight (body mass index (BMI) $\left.25-29.9 \mathrm{~kg} / \mathrm{m}^{2}\right)$ and obesity $(\mathrm{BMI} \geq 30 \mathrm{~kg} /$ $\mathrm{m}^{2}$ ) are causally linked to cardio-metabolic abnormalities like insulin resistance, dyslipidaemia and hypertension, which are major preventable risk factors for CVD. Latest figures show that $64 \%$ of adults in the UK are classified as overweight or obese. ${ }^{7}$ Overweight and obesity have not been consistently reported for people with SMI. A 2018 report based on a representative sample of English general practice records found that people with SMI were three times more likely to have obesity between the ages $15-34$, and 1.6 times more likely for ages 55-74 compared with the general population. ${ }^{8}$ However, these estimates were limited by high rates on noncompletion of BMI in general practice. Moreover, other estimates are often drawn from 
cross-sectional analyses and often include small numbers of people attending specific services within a geographical area with no comparison group. ${ }^{4}$

The factors underlying excess weight gain in people with SMI are multifactorial. Psychotropic drugs (ie, antipsychotics, antidepressants, mood stabilisers) are the mainstay treatment for the symptoms of psychosis and affect weight. ${ }^{9}$ Evidence from clinical trials has linked these drugs to rapid weight gain, but the long-term effects after a diagnosis are unclear. ${ }^{9}$ In one 11-year follow-up study of people with schizophrenia, increased CVD mortality was reported in people treated with quetiapine (adjusted HR: $1.41,95 \%$ CI 1.09 to 1.82 ) and reduced CVD mortality in people treated with clozapine (adjusted HR: 0.74, 95\% CI 0.60 to 0.91$).{ }^{10}$ However, these estimates may result from unmeasured confounding by socioeconomic and other demographic factors. ${ }^{11}$ Accordingly, a better understanding of the long-term impact of medication on weight change, cardio-metabolic risk factors, and incidence of and mortality from CVD is warranted.

Other factors that cause weight gain include unhelpful dietary patterns and physical inactivity. These behaviours are more common in people with SMI compared with the general population and may reflect the social disadvantage to which SMI contributes. ${ }^{12}$ In addition, the prevalence of smoking is estimated to be twice that in people with SMI compared with general population, ${ }^{13}$ and evidence shows people with schizophrenia smoke more cigarettes per day and more intensely than the general population of smokers. ${ }^{14}$ Other studies using polygenic risk scores for liability to schizophrenia have found no association between those scores and physical health outcomes except for conditions mediated through obesity and smoking. ${ }^{15}$ This suggests that the excess risk to people with SMI are modifiable.

Since obesity is modifiable, US and UK clinical guidelines recommend intervening to prevent and manage obesity. In the UK, the first-line intervention is general practitioner (GP) advice or a referral to a weight management programme to support an energy-restricted diet and increase physical activity for people with a BMI $>25$ $\mathrm{kg} / \mathrm{m}^{2}{ }^{16}{ }^{17}$ In the general population, trials of such programmes lead to greater weight-loss than self-directed attempts. ${ }^{18}$ Another study in the general population reported the rates of referral between 2005 and 2012 was 20.0 (95\% CI 19.3 to 20.8 ) per 1000 person-years, compared with weight-related advice at 30.3 (29.3 to 31.4). ${ }^{19}$ This rate is low, and although these interventions may not be recorded, other evidence also point to low rates of intervention. ${ }^{20}$ However, we have no data on whether GPs are using the annual review of people with SMI, which focuses specifically on preventative healthcare, to provide support for weight management such as referrals to weight management programme.

We have been unable to find studies that have followed people with SMI over several years to examine how weight and cardio-metabolic profiles change. It is possible these relate to ongoing psychotropic and/or weight management programmes. The longest study to date was an 18-month trial of people with schizophrenia. In this study, participants randomised to olanzapine had greater increases in weight, glycaemia and adverse lipid profile than other antipsychotics. ${ }^{21}$ Randomised controlled trials (RCTs) are often limited to short follow-up of selected populations, and there are methodological issues surrounding imputation strategies used to address high dropout rates. ${ }^{22}{ }^{23}$ Thus, it remains unclear how much weight and cardio-metabolic profiles change over the longer term, and how much of the excess incidence and mortality from CVD is explained by those changes in people with SMI.

In this protocol, we outline our plans to address this gap in a large, retrospective, population-based study in people with SMI matched with controls without SMI in the UK from 1998 to 2020. Our objectives are to:

- Examine the association between SMI and weight change.

- Examine the association between SMI and change in cardio-metabolic risk factors for CVD.

- Examine the association between SMI and incidence of and mortality from CVD.

- Compare incidence of advice and offer and actual referral to weight management services in people with both SMIs and BMI $\geq 25 \mathrm{~kg} / \mathrm{m}^{2}$ with controls.

\section{METHODS AND ANALYSIS}

\section{Patient and public involvement}

We consulted 12 members of the public with lived experience of SMI to ensure the outcomes that matter to them are included in the protocol. Their continued involvement will inform the dissemination of our results.

\section{Design}

This protocol is written according to the 'REporting of studies Conducted using Observational Routinelycollected health Data' statement. ${ }^{24}$ We will use a retrospective cohort using individual patient data from a large primary care database to examine all objectives. The planned study start date is 01 September 2021 and end date is 31 August 2024.

\section{Data source}

We will use the Clinical Practice Research Datalink (CPRD) Aurum-a governmental, not-for-profit research service holding anonymised primary care records drawn from participating general (primary care) practices dating back to 1987 (http://www.cprd.com). To date, it encompasses routinely collected patient data records of around 16 million patients who are currently registered with some 700 participating general practices. Patient demographic data (ie, year of birth, sex and ethnicity), characteristics (ie, height, weight, smoking status), symptoms, clinical diagnoses, consultations, referrals, prescribed medications and results of investigations are available. 


\section{Data linkage}

Data will be linked to the Hospital Episode Statistics (HES) and the Office for National Statistics mortality databases. Data linkage to the practice level Index of Multiple Deprivation (IMD), as a proxy measure for socioeconomic status (SES), will also be used when describing the baseline characteristics in the descriptive analyses and in secondary analyses.

\section{Population}

\section{Source population}

The source population will be selected from the CPRD Aurum. The source population will include all UK National Health Service (NHS) patients (male/female) registered with a participating general practice who are eligible for data linkage. We will not include up-tostandard records because this is not yet available as a measure of data quality in the CPRD Aurum database.

\section{Study cohort}

We will select a study cohort from the CPRD Aurum to investigate objectives $1-3$. We will identify and extract the records in the primary care and HES dataset for all patients with a diagnosis of SMI (hereafter exposed) and who are eligible for data linkage. Diagnostic Read codes, which are the standard clinical codes used in UK general practice, will be used to identify an SMI diagnosis. We will also use codes according to the International Classification of Diseases version 10 (ICD-10) from the HES data. The date of the first SMI Read code will be used as the index date, which is the earliest date that an exposed patient may enter the study. From the study cohort, a subcohort of patients will be selected to investigate objective 4 . To enter the subcohort, the exposed patient needs a recorded $\mathrm{BMI} \geq 25 \mathrm{~kg} / \mathrm{m}^{2}$ at any time after the index date. All information will be extracted for the period 01 January 1998 to 31 October 2020—the earliest and latest dates respectively that data are available.

\section{Exclusion criteria}

Patients will be excluded from the cohort(s) if they: have less than 1 year of active data in their participating practice before the index date; and/or who have a Read code for coronary heart disease, congestive heart failure or cardiomyopathy, and/or stroke before the index date. A flow diagram will present the selection and exclusion (with reasons where possible) of patients in the study.

\section{Duration of follow-up}

Patients will be followed up in the database from their index date until the earliest of the following: date of death; date of outcome of interest; date of leaving the participating practice; date of the latest download of data; or the end of the study follow-up period (ie, up to 22 years).

\section{Comparison group}

The unexposed represents the counterfactual and will include patients without an SMI Read code ever (hereafter unexposed) drawn from the source population. Up to four unexposed matches will be selected at baseline only if they have a qualifying weight Read code within \pm one year of the index date to the exposed in which they are matched. The matching variables include: age, sex, practice and calendar time. All weight Read codes will be extracted.

\section{Exposures}

For all analyses, the exposure of interest is SMI. We will extract information on the study cohort up to censoring. Extracted information includes the diagnosis date and type, which will be grouped according to the two major SMI subgroups as classified by the ICD-10: F20-29 nonaffective psychotic disorder (ie, schizophrenia, schizotypal and delusional disorders, psychosis not otherwise specified) and F30-39 affective psychotic disorder (ie, bipolar, manic, schizoaffective disorders, major depressive disorder with psychosis). Patients may have Read codes in the primary care data relating to both non-affective and affective psychotic disorders. These will be classified using the most recent Read code because we will assume that the latest diagnosis is the correct one. For example, it is common for diagnoses to be clarified over time from first episode psychosis to schizophrenia. Based on similar studies, instances where there are Read codes relating to both diagnoses at the same date will be coded under nonaffective psychotic disorder. ${ }^{25}$ Data on individual prescriptions for psychotropic medication will be extracted from the primary care data from index date until censored.

\section{Outcomes}

The primary aim of objective 1 is to assess the impact of an SMI diagnosis on weight change. A pilot search of the database indicated waist circumference was not reliably recorded so we did not include this as a measure of adiposity. The outcome for objective 2 is change in cardio-metabolic risk factors for CVD, which include change in systolic and diastolic blood pressure, change in total cholesterol/high-density lipoprotein ratio, and change in glycaemia indicated by HbAlc or fasting glucose. The outcome for objective 3 is incidence of and mortality from CVD. The objective for 4 is coded weight management advice and referral to weight management services. We will interpret the data based on the associations for all outcomes, as well as consistency and interpretable patterns. The specific outcomes for each objective are outlined in table 1 .

\section{Confounding}

We have identified confounding variables using directed acyclic graphs (DAGs), which aims to theoretically formalise potential confounders, colliders and biasing relationships. ${ }^{26}$ The DAG for each objective is described in online supplemental material S1. The DAG for objective 1 shows one direct path from the exposure (ie, SMI) to the outcome (ie, weight change), and four indirect paths: via health behaviours (ie, alcohol use, smoking and 
Table 1 Outcome measures

\begin{tabular}{|c|c|c|}
\hline Objective & Outcome & Measures \\
\hline \multirow{2}{*}{$\begin{array}{l}\text { Objective 1: Examine the association } \\
\text { between SMI and weight change }\end{array}$} & \multirow[t]{2}{*}{ Weight change } & Absolute mean weight change $(\mathrm{kg})$ \\
\hline & & Percentage weight change (kg) \\
\hline \multirow{5}{*}{$\begin{array}{l}\text { Objective 2: Examine the association } \\
\text { between SMI and changes cardio- } \\
\text { metabolic risk factors for CVD }\end{array}$} & \multirow[t]{5}{*}{ Cardio-metabolic risk } & Change in fasting blood glucose (FBG) \\
\hline & & Change in haemoglobin A1c (HbA1c) \\
\hline & & Change in triglycerides \\
\hline & & Change in low-density lipoprotein (LDL) \\
\hline & & $\begin{array}{l}\text { Change in blood pressure (systolic (SBP) and diastolic } \\
\text { (DBP)) }\end{array}$ \\
\hline \multirow[t]{4}{*}{ Objective 3: Total incident and fatal CVD } & \multirow{4}{*}{$\begin{array}{l}\text { Incidence of CVD and CVD } \\
\text { fatalities }\end{array}$} & Angina diagnosed by GP \\
\hline & & Cardiomyopathy \\
\hline & & Total stroke \\
\hline & & Total fatal CVD events \\
\hline $\begin{array}{l}\text { Objective } 4 \text { : Weight management advice } \\
\text { and service referral }\end{array}$ & \multicolumn{2}{|c|}{$\begin{array}{l}\text { Proportion (\%) of exposed and unexposed who are recorded as offered advice, and } \\
\text { then offered and actually referred to a weight management service }\end{array}$} \\
\hline
\end{tabular}

CVD, cardiovascular disease; GP, general practitioner; SMI, serious mental illness.

diet), SES status, psychotropic medication, and healthcare access and provision. Diet and physical activity are latent variables in CPRD Aurum because they are unmeasured. There are three confounding paths: via age, sex and ethnicity. ${ }^{27} 28$ Thus, the minimally sufficient adjustment for the total effect of SMI status on weight is to adjust for age, sex, and ethnicity.

Frequency matching will ensure our ability to adjust for confounding variables that include age, sex, and calendar year, and matching on practice will remove practice-specific variables. In addition, we will fully adjust our models by considering several covariates in the relationship between the exposure and outcome (see table 2). All categorical variables will be analysed using the natural reference category as the reference group. Data on individual prescriptions for psychotropic and cardio-metabolic medications will be extracted from the primary care data from index date until censoring. For psychotropic medications, drugs will be classified according to the British National Formulary. For objectives 2 and 3, we will assess the impact of weight gain on change in cardio-metabolic risk factors and incidence of CVD using mediation analysis to allow us to estimate the direct (unmediated) and indirect (mediated) effects of SMI on CVD risk and incidence. To do this, we will fit two regression models: one regressing the outcome on the exposure, adjusting for confounders and another regressing the mediator on the exposure and confounders using the Stata Med4way command to estimate mediated effects. ${ }^{29}$ For objective 4 , we will assess the presence of confounding by comparing estimates with and without adjustment. We will use Read codes, which have been previously developed by our team, to identify outcome and covariate records.

\section{Variable library}

Exposures, outcomes and covariates of interest will be identified using medical coding (Read/SNOMED/ EMIS), product coding $(\mathrm{DM}+\mathrm{D})$ and recording of patient death or transfer out of a participating practice in CPRD Aurum. The final definitions and code library has been drawn up and validated through consensus agreement among all investigators.

\section{Sample size calculation}

The estimated median follow-up time for the exposed with a weight Read code in their record in the 1 year before the index date is 3.2 years (mean: 4.6), which is based on our feasibility work for this project. We assumed a $1 \%$ annual incidence rate of CVD meaning $3 \%$ of the study population would develop CVD over the 3.2 years of follow-up. ${ }^{30}$ We also assumed a relative risk (RR) of 1.3 for CVD in the exposed versus unexposed. ${ }^{31}$ The $\mathrm{n}$ ratio was set at 0.25 , which achieved the best balance between statistical power and data cost. Our calculation suggested a minimum sample size of $\mathrm{n}=3907$ at $80 \%$ power to detect an RR of 1.3, alpha $\leq 0.05 \%$. Our feasibility estimates indicate that there are 135870 patients with SMI in CPRD Aurum, meaning the study is sufficiently powered to detect small effects. 


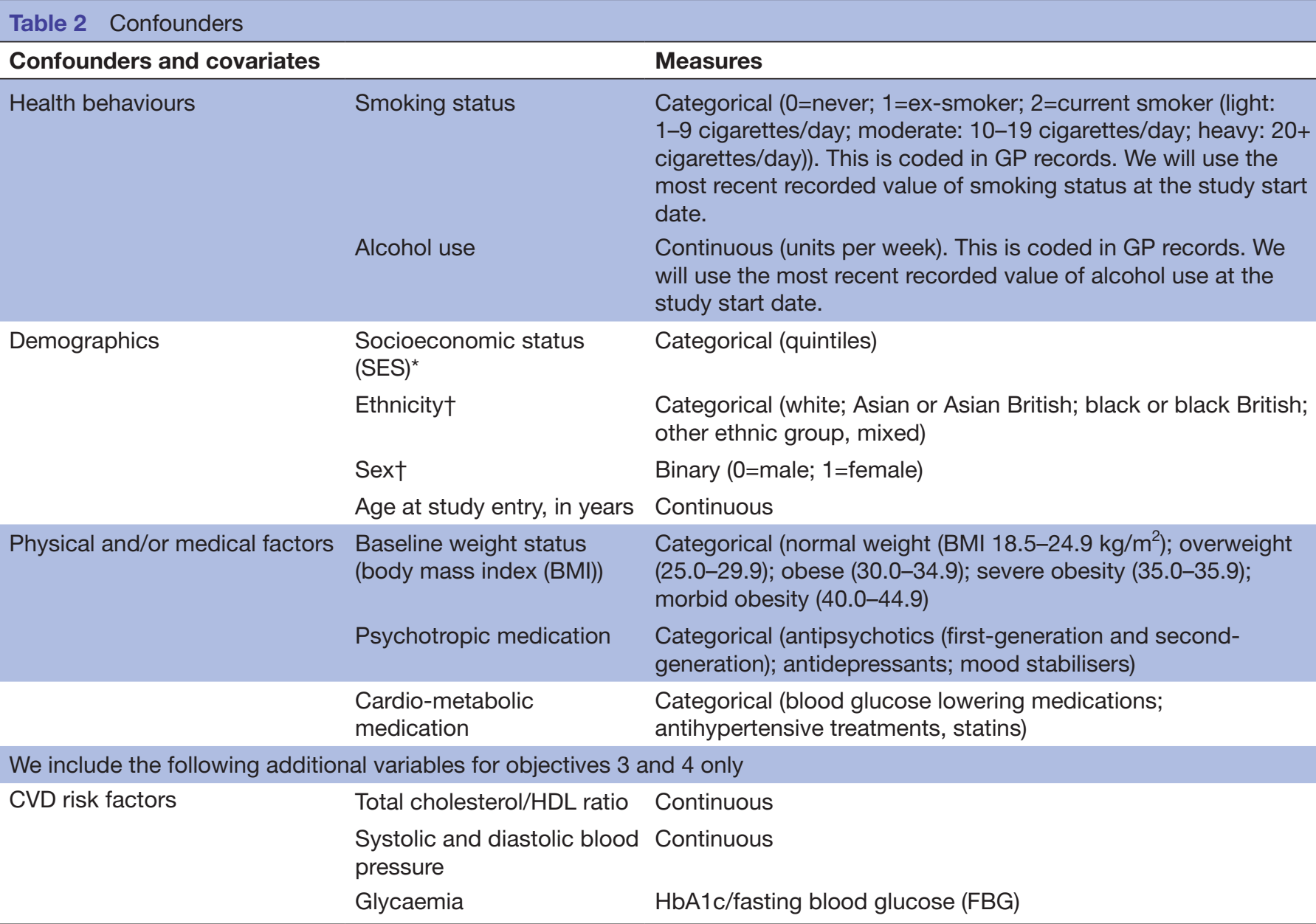

*This variable is grouped according to practice level Index of Multiple Deprivation. This is an indicator of material deprivation based on income; employment; health deprivation and disability; education, skills and training; crime; barriers to housing and services; and living environment. It is categorised as quintiles $1-5$ with 1 being the most deprived and 5 being the least deprived. †This variable is grouped according to recommendations by the UK Office for National Statistics. ${ }^{33} 34$ CVD, cardiovascular disease; GP, general practitioner; HbA1c, haemoglobin A1c; HDL, high-density lipoprotein.

\section{Statistical analysis}

Analyses will be conducted in Stata using an estimation approach. We do not intend to impute missing data. The entry date into the analysis is the index date. The outcome date is the earliest of: (1) the date of death or (2) date of diagnosis of the outcome of interest. For objectives 1 and 2, we will use all repeated measures and run separate models for each analyses. For objective 3, we will use the first recorded diagnosis of the outcome of interest rather than recurrent events. Patients who do not have the outcome of interest will be censored at the earliest of: (1) date of death, (2) date of leaving the practice, (3) date of the latest download of data or (4) the study end date.

\section{Descriptive summaries}

Frequency matching will reduce potential confounding and balance the baseline characteristics of the exposed and unexposed on age, sex, practice and calendar year. Adequacy of matching will be confirmed by comparing means using independent t-tests. Descriptive statistics will describe the baseline characteristics of patients in the cohort. Categorical data will be presented as frequency and percentages, and continuous variables will be summarised using descriptive statistics mean, standard deviation (SD) or median and interquartile range (IQR). Comparisons between the groups will be made using $\chi^{2}$ tests and t-tests (or Wilcoxon rank-sum tests) as appropriate. Length of exposure and the most commonly prescribed class of psychotropic for each patient will be summarised. The causes of death will be categorised as CVD and all-cause mortality.

\section{Primary analyses}

For objectives 1 and 2, the outcome will be visually inspected by plotting trajectories of weight and cardio-metabolic risk factor change using all repeated measurements for patients with SMI versus patients without SMI. For these analyses, we will use a mixed-effects linear regression model including terms for SMI, time and their interaction to estimate regression coefficients with $95 \%$ CIs by SMI status. Models incorporating psychotropic medications will analogously include medication-time multiplicative interaction terms. We will include a random effect for individual and practice identifier 
to account for intra-class correlation resulting from common practice membership. Diagnostic tests will be conducted to confirm linear regression assumptions are met including linearity of residuals, residual normality using quantilequantile $(\mathrm{Q}-\mathrm{Q})$ plots, and residual heteroscedasticity using residual versus fitted value plots. We will assess for exploratory subgroup effects by adding interactions (eg, by age, sex, ethnicity and SES) and, if significant, we will assess the strength of the associations in separate subgroups for clarity. For objective 2, we will model the association between weight and cardio-metabolic change using restricted cubic splines to allow for non-linear associations. For objective 3, we will use Kaplan-Meier survival estimates for the exposed and unexposed groups to estimate the distribution of time from index date to total and fatal CVD and mortality (all-cause). The statistical equivalence of the two curves will be examined using the log-rank test. This analysis will use a survival from index date between the exposed and unexposed groups using an adjusted Cox proportional hazards regression model with follow-up time as the timescale. Restricted cubic splines will be computed with five knots to visually explore non-linear associations between the exposure and the outcomes. For objective 4, we will use a Poisson regression examining person-time to estimate the rate at which patients who received weight management services over the study period in patients with SMI compared with patients who do not have SMI, adjusting for confounders and BMI.

\section{Sensitivity and secondary analyses}

If necessary, data on missing confounders and covariates will be addressed by adjusting for missingness as a category. Sensitivity analyses will examine the impact of running the analyses with everyone included as discussed above and confined only to those with complete data. A second sensitivity analysis will be performed excluding patients that experience the outcome within 2 years of the SMI Read code to avoid reversed causality from other underlying disease.

\section{ETHICS AND DISSEMINATION}

This study has been approved by the Independent Scientific Advisory Committee (ISAC) to the Medicines and Healthcare Products Regulatory Agency (reference: 20_000186). To guarantee the confidentiality of patient information, only the authors will have access to the data during the study. The full statistical code will be available from the authors after the publication of the results. The results of this study will be presented at scientific conferences and published in peer-reviewed journals.

\section{DISCUSSION}

In the UK, the NHS Long Term Plan commits the health service to greater action on reducing inequalities for the most overlooked groups. This study responds to this national agenda by focusing on the major cause of death for people with SMI-cardiovascular disease. We plan to leverage the power of a large UK primary care database to provide new insight on how these actions may be achieved.

Our results will estimate weight change, its risk factors including psychotropic medication, and its management in primary care as a preventative target for CVD. This could inform medication choice for people with SMI and clinicians, particularly if there are meaningful differences between drugs. In addition, our results will estimate the change in cardio-metabolic factors in people with SMI relative to those without SMI. This will be valuable to policy-makers because these are key risk factors for CVD that are being addressed through the NHS Long Term Plan. Furthermore, identifying the factors underlying the higher incidence of CVD will help us understand the degree to which these factors are valuable therapeutic targets. Lastly, understanding whether people with SMI are being offered support to manage their weight will inform national initiatives to overcome therapeutic nihilism. It is also important to understand whether there are differences in response between people with SMI and the general population, which would inform us about whether interventions need to be tailored to people with SMI for more personalised care. Ultimately, the results will offer a foundation to assess the degree to which the current focus on CVD prevention in people with SMI is having clinical benefits.

Since this study is observational, there are inherent strengths and limitations that are discussed. A strength of the study is its use of a representative, validated and sizeable dataset. Furthermore, the CPRD Aurum is an appropriate data source using primary care records. This will minimise selection, recall and respondent bias since UK general practices tend to have good levels of accuracy in recording prescriptions and clinical diagnoses. The results will also have direct and important applications to international guidelines on patient healthcare management, as well as to British primary care physicians, mental health services and members of the public with experience of SMI to initiate individual action. Finally, the longitudinal design is useful in addressing important clinical questions not usually possible within RCTs, and a matched-cohort improves efficiency since we will not need to include all controls, while tackling confounding.

Since the study is descriptive, any association observed would not imply an SMI diagnosis is causative of adverse physical health outcomes. However, we might assume the greater the magnitude of the observed association the less likely it is due to bias and more likely due to a causal process. $^{32}$ Furthermore, diet and physical activity are unmeasured confounders because they are unobserved in CPRD Aurum. Similarly, other unmeasured confounders like poverty may place people with SMI at increased risk of homelessness, which has consequences on healthcare access and provision. We address this potential confounding by linking to patient level IMD as a proxy measure of SES, but acknowledge this may not fully capture deprivation and leaves room for residual confounding. Moreover, information bias may be present since the CPRD Aurum relies on 
a GP recording of health outcomes. Furthermore, we can only examine primary care prescriptions. Any prescribing solely within secondary care may not be included as this was not historically routinely recorded in GP records. Thus, it is possible we will miss the first prescription(s). Finally, physical health checks are not always performed on random samples of the population, and it is possible that more regular testing is done on people with overweight and obesity. We expect that some patients will have incomplete information recorded. However, by determining what proportion of patients do not have appropriate information recorded, we can evaluate the scale of these problems, and highlight the need for improved recording for continuity of care between healthcare professionals.

\section{Twitter Felicity Waite @FelicityWaite}

Acknowledgements We thank the patient and public involvement volunteers for their time and for sharing their knowledge. We also thank the patients and practices who contribute to the CPRD database. We acknowledge that HES data that will be used in this analysis will be reused with permission from NHS Digital, who retain the copyright.

Contributors CL, FW, PA and CP conceived the study idea. CL, FW, MCS, MG, CB, $\mathrm{PA}$ and $\mathrm{CP}$ contributed to the development of the study design and protocol. $\mathrm{CL}$ drafted the manuscript. All authors approved the final submitted version.

Funding This research received no specific grant from any funding agency in the public, commercial or not-for-profit sectors. $\mathrm{CL}$ is funded by the Engineering and Physical Sciences Research Council (EPSRC; EP/R513295/1) and the National Institute for Health Research (NIHR) Oxford Biomedical Research Centre (BRC; IS-BRC-1215-20008). MCS is supported by the NIHR Oxford BRC. CB is supported by the NIHR Oxford BRC. PA is funded by the NIHR BRC, the NIHR ARC (200172), and is an NIHR senior investigator (NF-SI-0617-10064). CP is funded through the NIHR ARC. FW is funded by a Wellcome Trust Clinical Doctoral Fellowship (102176/B/13/Z).

Disclaimer The views expressed are those of the author(s) and not necessarily those of the NIHR or the Department of Health and Social Care.

Competing interests PA has conducted a trial funded by Cambridge Weight Plan and has spoken at a seminar at the Royal College of General Practitioners (RCGP) conference that was sponsored by Novo Nordisk.

Patient consent for publication Not applicable.

Provenance and peer review Not commissioned; externally peer reviewed.

Supplemental material This content has been supplied by the author(s). It has not been vetted by BMJ Publishing Group Limited (BMJ) and may not have been peer-reviewed. Any opinions or recommendations discussed are solely those of the author(s) and are not endorsed by BMJ. BMJ disclaims all liability and responsibility arising from any reliance placed on the content. Where the content includes any translated material, BMJ does not warrant the accuracy and reliability of the translations (including but not limited to local regulations, clinical guidelines, terminology, drug names and drug dosages), and is not responsible for any error and/or omissions arising from translation and adaptation or otherwise.

Open access This is an open access article distributed in accordance with the Creative Commons Attribution 4.0 Unported (CC BY 4.0) license, which permits others to copy, redistribute, remix, transform and build upon this work for any purpose, provided the original work is properly cited, a link to the licence is given, and indication of whether changes were made. See: https://creativecommons.org/ licenses/by/4.0/.

\section{ORCID iDs}

Charlotte Lee http://orcid.org/0000-0001-7115-5104

Felicity Waite http://orcid.org/0000-0002-2749-1386

Margaret C Smith http://orcid.org/0000-0002-0946-742X

Min Gao http://orcid.org/0000-0001-6196-7088

Paul Aveyard http://orcid.org/0000-0002-1802-4217

Carmen Piernas http://orcid.org/0000-0002-7536-922X
REFERENCES

1 England N. Improving physical healthcare for people living with severe mental illness (SMI) in primary care guidance for CCGs, 2018. Available: https://www.england.nhs.uk/wp-content/uploads/ 2018/02/improving-physical-health-care-for-smi-in-primary-care.pdf [Accessed 04 May 2021].

2 Hennekens CH, Hennekens AR, Hollar D, et al. Schizophrenia and increased risks of cardiovascular disease. Am Heart $J$ 2005;150:1115-21.

3 Osborn DPJ, Levy G, Nazareth I, et al. Relative risk of cardiovascular and cancer mortality in people with severe mental illness from the United Kingdom's general practice Rsearch database. Arch Gen Psychiatry 2007;64:242-9.

4 Correll CU, Solmi M, Veronese N, et al. Prevalence, incidence and mortality from cardiovascular disease in patients with pooled and specific severe mental illness: a large-scale meta-analysis of $3,211,768$ patients and $113,383,368$ controls. World Psychiatry 2017;16:163-80.

5 Yusuf S, Reddy S, Ôunpuu S, et al. Global burden of cardiovascular diseases. Circulation 2001;104:2746-53.

6 Saha S, Chant D, McGrath J. A systematic review of mortality in schizophrenia: is the differential mortality gap worsening over time? Arch Gen Psychiatry 2007;64:1123-31.

7 NHS Digital. Health Survey for England 2019 [NS] - NHS Digital. Available: https://digital.nhs.uk/data-and-information/publications/ statistical/health-survey-for-england/2019\# [Accessed 30 Sep 2021].

8 National Mental Health Intelligence Network. Severe mental illness (SMI) and physical health inequalities: briefing - GOV.UK. Public Health England, 2018Published. Available: https://www.gov.uk/ government/publications/severe-mental-illness-smi-physical-healthinequalities/severe-mental-illness-and-physical-health-inequalitiesbriefing\#fn:1 [Accessed 14 Nov 2019].

9 Leslie WS, Hankey CR, Lean MEJ. Weight gain as an adverse effect of some commonly prescribed drugs: a systematic review. QJM 2007;100:395-404.

10 Tiihonen J, Lönnqvist J, Wahlbeck K, et al. 11-Year follow-up of mortality in patients with schizophrenia: a population-based cohort study (FIN11 study). Lancet 2009;374:620-7.

11 De Hert M, Correll CU, Cohen D. Do antipsychotic medications reduce or increase mortality in schizophrenia? A critical appraisal of the FIN-11 study. Schizophr Res 2010;117:68-74.

12 Teasdale SB, Ward PB, Samaras K, et al. Dietary intake of people with severe mental illness: systematic review and meta-analysis. $\mathrm{Br} \mathrm{J}$ Psychiatry 2019;214:251-9.

13 Psychiatrists RC of PRC of. Smoking and mental health. London, UK, 2013.

14 Williams JM, Ziedonis DM, Abanyie F, et al. Increased nicotine and cotinine levels in smokers with schizophrenia and schizoaffective disorder is not a metabolic effect. Schizophr Res 2005;79:323-35.

15 Kendall KM, John A, Lee SC, et al. Impact of schizophrenia genetic liability on the association between schizophrenia and physical illness: data-linkage study. BJPsych Open 2020;6:e139.

16 Agency for Healthcare Research and Quality. U.S. preventive services Task force. Rockville, MD, 2019. Available: https://www. ahrq.gov/cpi/about/otherwebsites/uspstf/index.html

17 The National Institute for Health and Care Excellence (NICE). Obesity prevention clinical guideline [CG43]. United Kingdom, 2015. Available: https://www.nice.org.uk/guidance/CG43

18 Jolly $\mathrm{K}$, Lewis $\mathrm{A}$, Beach J, et al. Comparison of range of commercial or primary care led weight reduction programmes with minimal intervention control for weight loss in obesity: Lighten up randomised controlled trial. BMJ 2011;343:d6500.

19 Booth HP, Prevost AT, Gulliford MC. Access to weight reduction interventions for overweight and obese patients in UK primary care: population-based cohort study. BMJ Open 2015;5:e006642.

20 Humphreys K, Blodgett JC, Roberts LW. The exclusion of people with psychiatric disorders from medical research. J Psychiatr Res 2015;70:28-32.

21 Lieberman JA, Stroup TS, McEvoy JP, et al. Effectiveness of antipsychotic drugs in patients with chronic schizophrenia. $N$ Engl J Med 2005;353:1209-23.

22 Jones PB, Barnes TRE, Davies L, et al. Randomized controlled trial of the effect on quality of life of second- vs first-generation antipsychotic drugs in schizophrenia: cost utility of the latest antipsychotic drugs in schizophrenia study (CUtLASS 1). Arch Gen Psychiatry 2006;63:1079-87.

23 Alvarez-Jiménez M, González-Blanch C, Crespo-Facorro B, et al. Antipsychotic-Induced weight gain in chronic and first-episode psychotic disorders: a systematic critical reappraisal. CNS Drugs 2008;22:547-62. 
24 Benchimol El, Smeeth L, Guttmann A, et al. The reporting of studies conducted using observational Routinely-collected health data (record) statement. PLoS Med 2015;12:e1001885.

25 Hope H, Parisi R, Ashcroft DM, et al. Fertility trends of women with serious mental illness in the United Kingdom 1992-2017: a primary care cohort study using the clinical practice research Datalink. $J$ Affect Disord 2020;269:141-7.

26 Shrier I, Platt RW. Reducing bias through directed acyclic graphs. BMC Med Res Methodol 2008;8:70.

27 Merikangas KR, He J-P, Burstein M, et al. Lifetime prevalence of mental disorders in U.S. adolescents: results from the National Comorbidity Survey Replication--Adolescent Supplement (NCS-A). J Am Acad Child Adolesc Psychiatry 2010;49:980-9.

28 Schwartz RC, Blankenship DM. Racial disparities in psychotic disorder diagnosis: a review of empirical literature. World J Psychiatry 2014;4:133.
29 Discacciati A, Bellavia A, Lee JJ, et al. Med4way: a Stata command to investigate mediating and interactive mechanisms using the fourway effect decomposition. Int J Epidemiol 2019;48:15-20.

30 Fan Z, Wu Y, Shen J, et al. Schizophrenia and the risk of cardiovascular diseases: a meta-analysis of thirteen cohort studies. $J$ Psychiatr Res 2013;47:1549-56.

31 Cunningham R, Poppe K, Peterson D, et al. Prediction of cardiovascular disease risk among people with severe mental illness: a cohort study. PLoS One 2019;14:e0221521.

32 Grimes DA, Schulz KF. Bias and causal associations in observational research. Lancet 2002;359:248-52.

33 Government Statistical Service. Ethnicity harmonised standard, 2019. Available: https://gss.civilservice.gov.uk/policy-store/ethnicity/ [Accessed 13 Aug 2021].

34 Government Statistical Service. Sex and gender harmonisation guidance, 2019. Available: https://gss.civilservice.gov.uk/policystore/sex-and-gender/ [Accessed 13 Aug 2021]. 